\title{
Forward Link Performance Analysis for the Future IEEE 802.16-based Airport Data Link
}

\author{
Paola Pulini \\ Institute of Communication and Navigation \\ Deutsches Zentrum für Luft- und Raumfahrt (DLR) \\ 82234 Wessling, Germany \\ Email: Paola.Pulini@dlr.de
}

\begin{abstract}
In this paper, an analysis of the performance of the OFDM version of the IEEE 802.16e standard is provided in a realistic airport scenario. We implemented a novel stochastic airport channel model describing the main wireless propagation phenomena related to the airport environment. Multi-path and Doppler effects with the potentially resulting inter-symbol interference and inter-carrier interference have been taken into account, respectively. System performance is evaluated for the forward link case in the apron, parking, taxi and runway scenarios, showing strengths and lacks of the system characteristics. For the most critical scenario (i.e., the parking one), an investigation of the reduction of the pilot sub-carriers spacing and on the introduction of antenna diversity is proposed, showing large performance gains.
\end{abstract}

\section{INTRODUCTION}

The scope of this investigation deals with the development of technologies able to support the increasing data traffic coming from vehicles operating on the airport surface, i.e. mainly aircraft, but also vehicles providing luggage handling, fueling, etc. Currently, for the air traffic data link communications, only the highly congested very high frequency (VHF)-band is used. Moreover, the VHF Digital Link Mode 2 (VDL2) [1] technology, which is currently adopted, cannot satisfy the demand of robustness, security and efficiency required for future aeronautical communications. New bands and new data communication systems have to be investigated. For these reasons, at the World Radio Conference in 2007 [2], new allocations to the aeronautical communications were defined, especially in $\mathrm{C}$ band (between $5.091 \mathrm{GHz}$ and $5.150 \mathrm{GHz}$ ) for airport surface operations. The standard IEEE 802.16e (WiMAX) [3] [4] has been recommended by the European Organization for the Safety of Air Navigation (EUROCONTROL) and the Federal Aviation Administration (FAA) as the future airport data link technology. The WiMAX standard provides a large variety of profiles for physical layer coding and modulation, and hence enables the choice of the one which may be most suitable for the airport environment. The standard includes three different types of physical layer, one based on single-carrier transmission and the other two on multi-carrier transmission, namely on orthogonal frequency-division multiplexing (OFDM) and orthogonal frequency-division multiple-access (OFDMA), respectively. The multi-carrier modes offer a strong resistance against the multi-path effects. Hence, they represent an appealing solution for the airport scenarios, which are characterized by frequent multi-path propagation conditions.

To determine the best solution for the airport data link, a good knowledge of the characteristics of the communication channel is extremely important. The airports differ one to each other for size and structure. Nevertheless, they are all characterized by the presence of typical communication scenarios. Generally, it is possible to distinguish four different areas with different propagation conditions. The four areas are referred to as apron, taxi, parking and runway. The apron scenario represents the area in front of the terminals, with line-of-sight (LOS) condition for most of the time and limited aircraft mobility $(\sim 30 \mathrm{~km} / \mathrm{h})$. The parking area is also close to the buildings, but here the control tower is in non line-ofsight (NLOS) condition with the vehicles for most of the time. In this scenario the aircraft have very low speeds or are not moving at all. The taxi scenario represents the phase during which the aircraft is traveling toward or from the gate. Speeds of $50-60 \mathrm{~km} / \mathrm{h}$ are typical, as well as having LOS component. Finally, the runway scenario is similar to the taxi case, but shows considerably higher speeds.

In this paper we propose to investigate the performance of the OFDM mode of the WiMAX standard for the airport data link, focusing on the forward link (FL) ${ }^{1}$ case. In order to provide a realistic performance analysis of the airport area, we used a stochastic channel model with parameters derived from a recent measurement campaign at Munich airport [5].

Section II is dedicated to a description of the channel model used, its implementation and the parameters chosen for each scenario. Section III provides a description of the system, summarizing the main physical layer characteristics of the OFDM mode of the WiMAX standard and the parameters used for the simulations. Section IV provides simulation results obtained in the four different airport scenarios. Moreover, for the most critical scenario (parking) performance improvements through a pilot spacing reduction and the addition of antenna diversity are evaluated. Section V concludes this work, summarizing the main outcomes for the different airport scenarios and identifying strengths and the lacks of the evaluated system.

\footnotetext{
${ }^{1}$ In the aeronautical context, the FL is usually referred to as the link from the control tower to the aircraft, while the reverse link (RL) represents the link from the aircraft to the control tower.
} 


\section{Channel MOdel Description}

The channel adopted for the performance analysis is based on the wide sense stationary uncorrelated scattering (WSSUS) model [6], adapted to the peculiarities of the airport environment. The model implementation permits to evaluate the effects related to Doppler spread and multi-path on the reception of the OFDM signal. Hence, inter-symbol interference (ISI) and inter-carrier interference (ICI) will be considered in the next sections. The multi-path phenomena are implemented through a tapped delay line with $N_{t}$ taps. Moreover, the fading processes are independent among taps. Each tap is characterized by an amplitude $c_{k}, k=1 \ldots N_{t}$, a delay $\tau_{k}$ and a discrete-time correlated fading process $\alpha_{k}\left(n T_{c}\right)$, with $T_{c}$ being the sampling period of the received signal. As shown in Figure 1, the received signal $y\left(n T_{c}\right)$ results from the sum of all the tap components, obtained by multiplying the delayed transmitted signal $x\left(n T_{c}\right)$ by the different fading coefficients $\alpha_{k}\left(n T_{c}\right)$. The received signal is hence given by

$$
y\left(n T_{c}\right)=\sum_{k=0}^{N_{t}-1} x\left(n T_{c}-\tau_{k}\right) \cdot \alpha_{k}\left(n T_{c}\right),
$$

where $n T_{c}$ represents the generic $n^{\text {th }}$ sample time. The amplitude coefficients $c_{k}$ and the tap delays $\tau_{k}$ are obtained according to an exponentially decaying power delay profile.

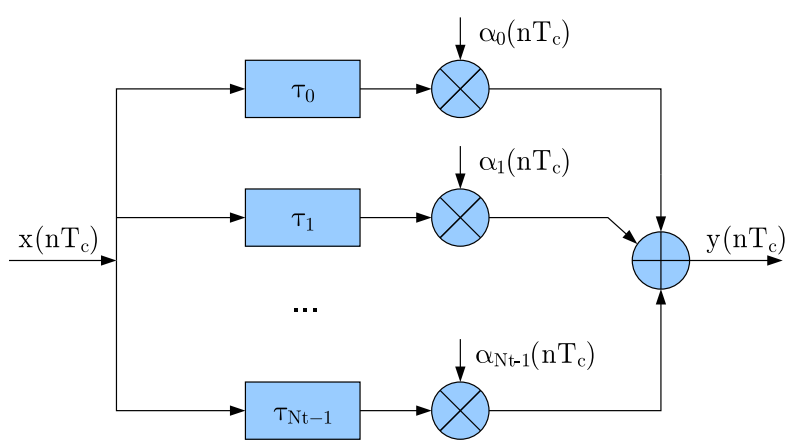

Fig. 1. Time domain multi-path channel model scheme.

The fading coefficients are given by samples of correlated Rayleigh and Rice processes, generated with the sum of sinusoids method [7]-[9], which obtains the fading by superposing a finite number of scatterers. The $k^{t h}$ tap coefficient is provided by

$$
\begin{gathered}
\alpha_{k}\left(n T_{c}\right)=c_{k}\left(n T_{c}\right) \cdot \sqrt{\frac{1}{N_{s}}} \sum_{l=0}^{N_{s}-1} e^{j\left[2 \pi\left(f_{l, k}+f_{d_{k}}\right) n T_{c}+\theta_{l, k}\right]}, \\
k=0, \ldots, N_{t}-1, \quad n=0,1,2, \ldots
\end{gathered}
$$

The fading amplitude is given by the tap gain $c_{k}$ and by a normalization coefficient, inversely proportional to the number of scatterers $N_{s}$. The phase $\theta_{l, k}$ is chosen as a random variable uniformly distributed between 0 and $2 \pi$ while the frequency is given by the sum of the Doppler frequency shift $f_{d_{k}}$ of the $k^{t h}$ tap, and a variable Doppler frequency $f_{l, k}$, following a certain power spectral density (PSD) distribution depending on the different scenario characteristics. Figure 2 describes the generation of a fading coefficient.

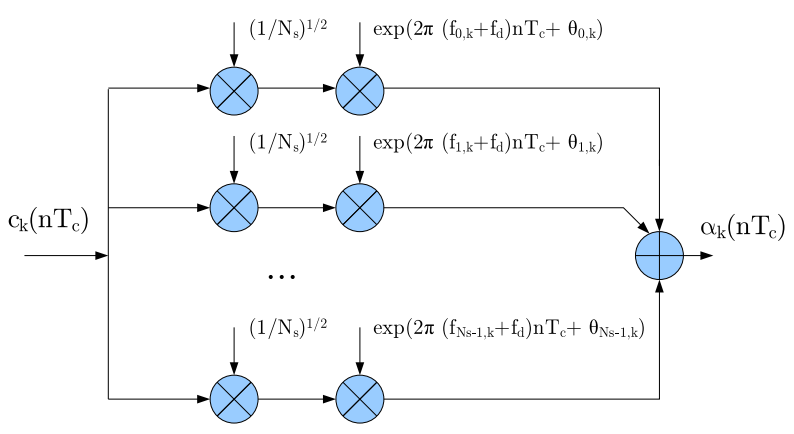

Fig. 2. Fading generation scheme with the sum of sinusoids method.

For the LOS component (tap $k=0$ ), the fading coefficients $\alpha_{0}\left(n T_{c}\right)$ are given by

$$
\alpha_{0}\left(n T_{c}\right)=c_{0}\left(n T_{c}\right) \cdot \sqrt{\frac{1}{N_{s}}} e^{j\left(2 \pi f_{d_{0}} n T_{c}\right)},
$$

i.e., the LOS component is affected by a rigid Doppler shift only. This model permits to calculate the impact of the ICI phenomenon on the OFDM system performance. We have chosen the Doppler shift $f_{d_{k}}$ values uniformly distributed in $\left[-f_{d_{\max }}, f_{d_{\max }}\right]$, with

$$
f_{d_{\max }}=\frac{f_{c} \cdot v}{c},
$$

where $f_{c}$ represents the carrier frequency, $v$ is the speed of the aircraft and $c=3 \cdot 10^{8} \mathrm{~m} / \mathrm{s}$ is the speed of the light. The Doppler frequencies for the scatterers have been selected by means of the Monte Carlo method [10]-[12] according to a Gaussian PSD (GPSD). Theoretical investigations, in fact, showed that aeronautical channels are characterized by Gaussian-shaped Doppler spectrum [13].

\section{A. Channel Parameters}

The channel model parameters have been selected according to a measurement campaign carried out at Munich airport [5]. Although each airport has its own size, structure, geographic configuration and therefore an own propagation environment, it is possible to assume the results coming from a typical airport as approximation for other airports of similar size. All airports include limited mobility scenarios, as apron and parking, and higher mobility ones, as taxi and runway. Each scenario presents similar propagation conditions. For a single scenario, characteristic values may be extrapolated from one single case as representative of other cases.

The power of the strongest component changes considerably between different airport areas, but is subjected to variations within a scenario. Therefore the determination of a unique Rice factor $(K)$ characterizing the scenario becomes hard task. For this reason we decided to consider few characteristic values for each scenario. For the scenarios with frequent LOS 
component we chose $K$ equal to 0,10 and $20 \mathrm{~dB}$, while for the scenarios where the NLOS condition is predominant, we selected $K=0$ and $K=-10 \mathrm{~dB}$.

We adopted an exponentially decaying power delay profile with delay spreads $\sigma_{\tau}$ chosen as the $99^{t h}$ percentile of the cumulative distribution function (CDF) of $\sigma_{\tau}$ obtained from the measurement results [5]. The number of taps $N_{t}$ comes from [5] as well.

The Doppler frequency shift $f_{d}$ follows an uniform distribution between the minimum and maximum values $-f_{d_{\max }}$ and $f_{d_{\max }}$. The maximum Doppler shifts given in Table I have been determined according to typical speed profiles. For instance, for the apron scenario, the typical speed is in the order of $30 \mathrm{~km} / \mathrm{h}$. Assuming a carrier frequency $f_{c}=5$ $\mathrm{GHz}$, the $f_{d_{\max }} \simeq 140 \mathrm{~Hz}$, for all $k=0 \ldots N_{t}-1$. All the fading components have a Gaussian shaped Doppler spectrum but the LOS component. We used Doppler spread values $\sigma_{f_{D}}$ distributed between the minimum ad maximum values indicated in Table I. The number of scatterers $N_{s}=25$ assures a good compromise between sufficiently good spectrum shape approximation and simulation complexity.

TABLE I

CHANNEL PARAMETERS USED FOR THE DIFFERENT SCENARIOS.

\begin{tabular}{|l||c|c|c|c|}
\hline & APR & PARK & TX & RWY \\
\hline \hline $\mathrm{K}[\mathrm{dB}]$ & $0,10,20$ & $0,-10$ & $0,10,20$ & $0,10,20$ \\
\hline$\sigma_{\tau}[\mu \mathrm{s}]$ & 0.65 & 1.25 & 1.5 & 1.05 \\
\hline$\sigma_{f_{D \min }, \sigma_{f_{D \max }}[\mathrm{Hz}]}$ & 20,50 & 10,40 & 40,95 & 100,200 \\
\hline$f_{d \max }[\mathrm{Hz}]$ & 140 & 50 & 150 & 500 \\
\hline$N_{t}$ & 9 & 12 & 6 & 7 \\
\hline$N_{s}$ & 25 & 25 & 25 & 25 \\
\hline
\end{tabular}

\section{SySTEM CHARACTERISTICS}

We evaluated the performances of a system based on the OFDM mode of the IEEE 802.16e (WiMAX) standard [4] in the FL context. The OFDM waveform of the WiMAX standard consists of 256 sub-carriers, out of which only 200 (in the center of the bandwidth) are effectively used for data transmission and for pilot symbols (except for the DC subcarrier, which is nulled). Two lateral guard-bands of 27 and 28 sub-carriers are left unused. The signal bandwidth is adjusted by scaling the sub-carrier spacing $\Delta_{f}$ according to the needs. The maximum bandwidth allocation is set to $5 \mathrm{MHz}$. The fixed distance between two pilot sub-carriers $\Delta_{p}$ is equal to $25 \Delta_{f}$ for all the symbols, as shown in Figure 3.

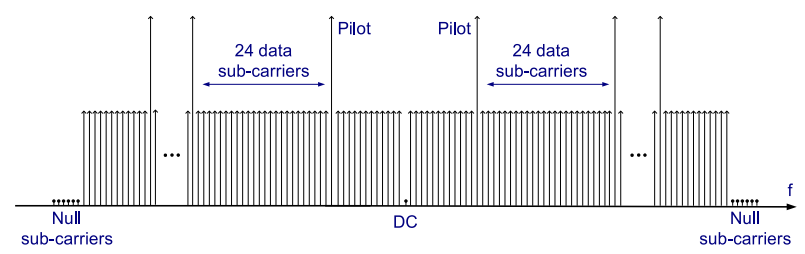

Fig. 3. Symbol structure for the OFDM case

The basic coding scheme for this mode is based on the concatenation of an outer Reed-Solomon code with an inner convolutional code (RS-CC) with different coding rates.
Optional codes are the convolutional turbo code (CTC) and block turbo code (BTC). The modulation set for the subcarriers includes binary phase shift keying (BPSK), quadrature phase shift keying (QPSK), quadrature amplitude modulation (QAM) with 16 constellation points (16-QAM) and optionally 64-QAM. The cyclic prefix may be selected within $1 / 4,1 / 8$, $1 / 16$ or $1 / 32$ of the symbol duration.

\section{A. System Parameters}

The parameters chosen for the simulations consist of a bandwidth of $5 \mathrm{MHz}$ with subcarrier spacing of roughly 20 $\mathrm{kHz}$, a basic coding scheme with a rate $1 / 2$ convolutional code and a QPSK subcarrier modulation. Given this subcarrier spacing and the Doppler spread/shift values presented in Section II-A, the inter-carrier interference shall provide a negligible impact on the performance. The cyclic prefix has been set $1 / 8$ of the symbol length, therefore the total OFDM symbol time $T_{\text {OFDM }}$ is approximatively $57.6 \mu \mathrm{s}$. Frames of 24 OFDM symbols have been considered, with all available sub-carriers allocated to one user. Prior to the modulation, the bits at the output of the convolutional encoder are interleaved over the entire frame. At the receiver side we implemented a linear channel interpolation in the frequency domain, using the pilot tones. Table II provides the system parameters used in the simulations.

TABLE II

SIMULATION SYSTEM PARAMETERS.

\begin{tabular}{|l||c|}
\hline System parameters & FL - OFDM \\
\hline \hline Bandwidth & $5 \mathrm{MHz}$ \\
\hline FFT size & 256 \\
\hline Null guard sub-carriers & 56 \\
\hline Pilot sub-carriers/ OFDM symbol & 8 \\
\hline Cyclic prefix $(\mathrm{CP})$ & $1 / 8 \cdot T_{S}=6.4 \mu \mathrm{s}$ \\
\hline Frame size & 24 OFDM symbols \\
\hline Symbol time $\left(T_{S}\right)$ & $51.2 \mu \mathrm{s}$ \\
\hline$T_{\text {OFDM }}=T_{S}+\mathrm{CP}$ & $57.6 \mu \mathrm{s}$ \\
\hline$T_{\text {frame }}$ & $1.38 \mathrm{~ms}$ \\
\hline Sub-carrier spacing $\Delta_{f}$ & $\simeq 20 \mathrm{kHz}$ \\
\hline Pilot sub-carrier spacing $\Delta_{p}$ & $25 \Delta_{f} \simeq 500 \mathrm{kHz}$ \\
\hline Coding, rate & Convolutional, $1 / 2$ \\
\hline Decoding & Soft Viterbi \\
\hline Modulation & QPSK \\
\hline Pilot Boosting & $2.5 \mathrm{~dB}$ \\
\hline Channel Estimation & Linear Interpolation \\
\hline
\end{tabular}

\section{Simulation RESUlts}

In the following we present the simulation results, evaluating the performance of the above-described system on the channel described in Section II. The results are presented separately for each scenario and are shown in terms of bit error rate (BER) versus $E_{b} / N_{0}$.

In Figure 4, the results obtained for the apron scenario are provided. This case is characterized by a delay spread $\sigma_{\tau}=$ $0.65 \mu \mathrm{s}$ and small values of Doppler spread and shift $\left(\sigma_{f_{D}} \leq\right.$ $50 \mathrm{~Hz},\left|f_{D}\right|<140 \mathrm{~Hz}$ ). The plots obtained with the higher $K$ values are encouraging while the one obtained with $K=0$ $\mathrm{dB}$ presents a relatively high error floor. 


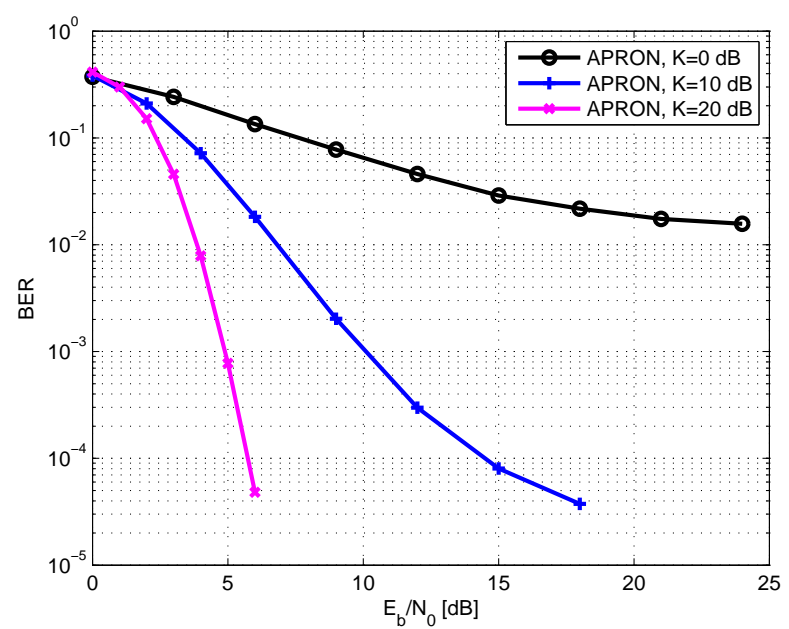

Fig. 4. Apron scenario performances with respect to different Rice factor values $(\mathrm{K}=0,10$ and $20 \mathrm{~dB}) . \sigma_{\tau}=0.65 \mu \mathrm{s}, \sigma_{f_{D}} \leq 50 \mathrm{~Hz}$ and $f_{d}$ uniformly distributed between $(-140,140) \mathrm{Hz}$.

The parking scenario corresponds to the lowest mobility case with NLOS conditions for most of the time. This scenario is characterized by a rich multi-path with a large delay spread, while the low mobility results in small Doppler spreads/shifts. Rice factor values of 0 and $-10 \mathrm{~dB}$ have been chosen to reproduce the predominance of the fading components. Performances provided by Figure 5 show that the lack of LOS component plays a dominant role. The performance is very poor and high error floors are present.

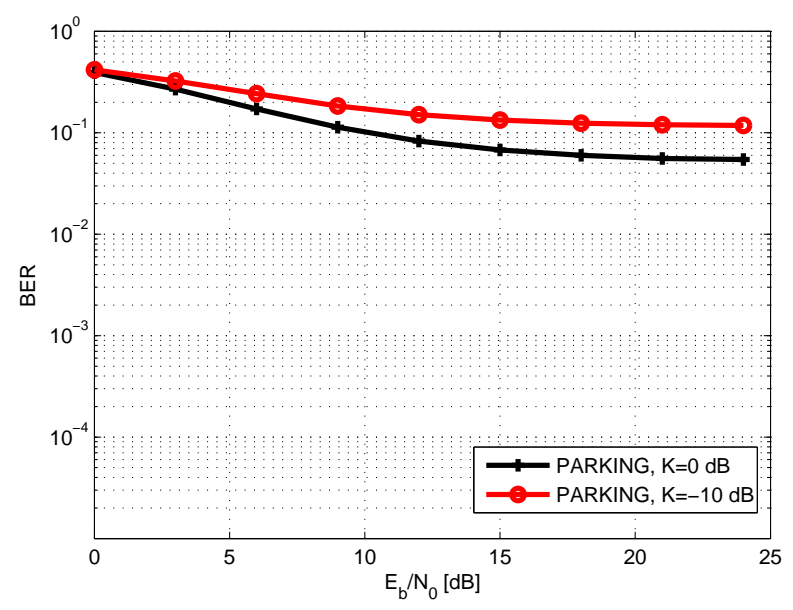

Fig. 5. Parking scenario performances with respect to different Rice factor values $(\mathrm{K}=0$ and $-10 \mathrm{~dB}), \sigma_{\tau}=1.25 \mu \mathrm{s}, \sigma_{f_{D}} \leq 40 \mathrm{~Hz}$ and $f_{d}$ uniformly distributed between $(-50,50) \mathrm{Hz}$.

Figure 6 provides the results obtained for the taxi scenario. Here, respect to the previous cases, there is more mobility and typically LOS condition, which reflects on the relatively good performance for $K=10 \mathrm{~dB}$ and $K=20 \mathrm{~dB}$. Vice versa, the less characteristic $K=0 \mathrm{~dB}$ chart, included for completeness, presents poor performance with the highest error floors w.t.r. the other scenarios.

Figure 7 shows the performance obtained for the runway area. This scenario presents the highest mobility and the highest Doppler values, though the large sub-carrier spacing of $20 \mathrm{kHz}$ assures no appreciable degradation due to ICI. Also

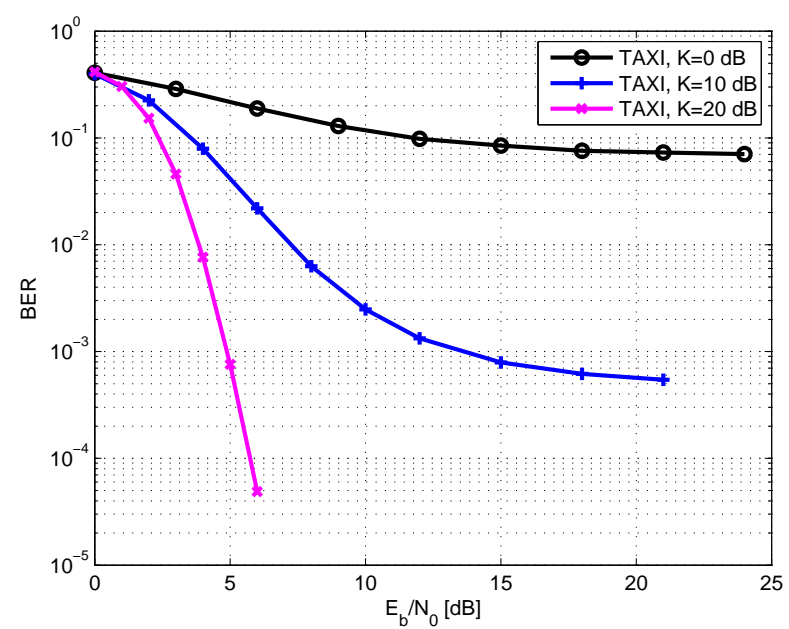

Fig. 6. Taxi scenario performances with respect to different Rice factor values $(\mathrm{K}=0,10$ and $20 \mathrm{~dB}) . \sigma_{\tau}=1.5 \mu \mathrm{s}, \sigma_{f_{D}} \leq 95 \mathrm{~Hz}$ and $f_{d}$ uniformly distributed between $(-150,150) \mathrm{Hz}$.

in this scenario the LOS component is predominant, hence the Rice factor is realistically in the range $10-20 \mathrm{~dB}$. This scenario presents indeed the best performance.

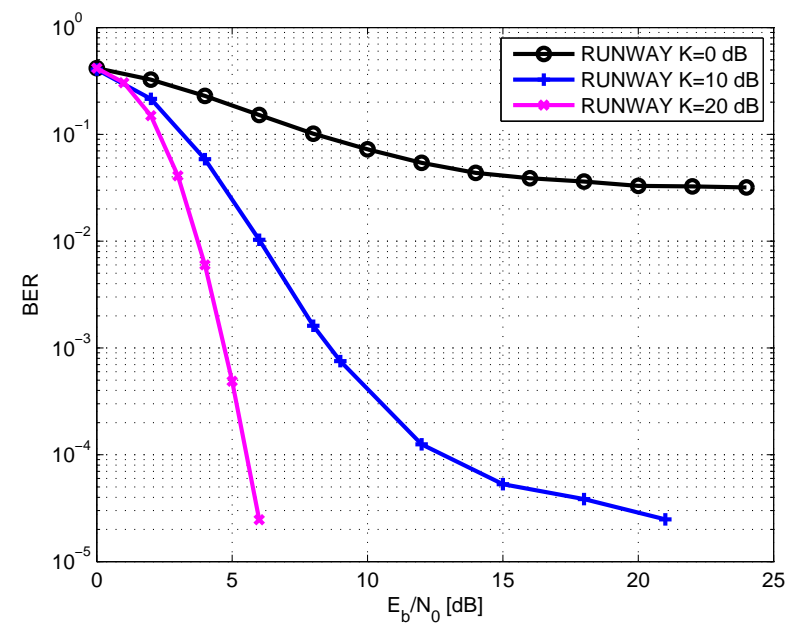

Fig. 7. Runway scenario performances with respect to different Rice factor values $(\mathrm{K}=0,10$ and $20 \mathrm{~dB}) . \sigma_{\tau}=1.05 \mu \mathrm{s}, \sigma_{f_{D}} \leq 200 \mathrm{~Hz}$ and $f_{d}$ uniformly distributed between $(-500,500) \mathrm{Hz}$.

In general in LOS conditions performances are sufficiently good for all the scenarios. The small differences within the scenarios may be explained considering the different delay spreads and Doppler values.

In case of Rice factor values $\leq 0 \mathrm{~dB}$, vice versa, the performances of the system are generally particulary poor. Considering that the cyclic prefix $\mathrm{CP}=0.64 \mu \mathrm{s}$ is sufficient to avoid ISI and that the sub-carrier spacing of $20 \mathrm{kHz}$ should assure no ICI, this behavior can be explained considering a lack of time selectivity and, most of all, an inaccurate channel estimation. The distance between two pilot sub-carriers $\left(\Delta_{p}=25 \Delta_{f}\right)$ is too large for the frequency selectivity of the channel. Therefore, the channel behavior cannot properly be tracked. The inefficient channel estimation causes the error floors and jeopardizes any eventual frequency diversity effect. Indeed, the worst performances correspond to the highest delay 
spread values, i.e. the taxi $\left(\mu_{\tau}=1.5 \mu s\right)$ and the parking $\left(\mu_{\tau}=1.25 \mu s\right)$ scenarios.

We hence decided to investigate simple techniques for enhancing the system performance, with particular emphasis to the parking scenario, which presents the major performance degradation. As discussed before, the two main issues affecting the system performance are the too large separation between pilot sub-carriers in the frequency domain and the limited time diversity provided by the channel. The problem has been thus tackled from both sides. On one hand a basic $1 \times 2$ single input multiple output (SIMO) scheme (with two antennas at the receiver side) has been introduced to enhance the diversity. On the other hand, increased pilot sub-carrier densities have been investigated to allow accurate channel estimation even in presence of large delay spreads. In Figure 8, the results obtained by reducing the pilot sub-carrier spacing with and without the $1 \times 2$ SIMO scheme are presented. The results are related to a parking scenario with a Rice factor $K=-10 \mathrm{~dB}$. When the SIMO scheme is adopted, maximum ratio combining (MRC) of the received signals is performed, using the channel estimates provided by the linear interpolator. Interestingly, the introduction of the second antenna, leaving the pilot sub-carrier spacing to $25 \Delta_{f}$, brings a moderate performance improvement. The error floor is slightly lowered, but it still appears at rather high error rates $\left(\mathrm{BER} \simeq 10^{-2}\right.$ ). This phenomenon is due to the insufficient carrier spacing w.r.t. to the frequency selectivity introduced by the relatively high delay spread. We thus investigated the performance of the system with an increased pilot density, i.e. with pilot sub-carrier spacing $10 \Delta_{f}$ and $5 \Delta_{f}$. The framing has been modified accordingly, preserving the lateral null guard bands. The results are shown in Figure 8 and confirm our conjecture. In particular, the reduction of the sub-carrier spacing to $5 \Delta_{f}$ permits to remove the floors (at least, down to the error rates achieved through the simulations) and permits to highlight the diversity gain provided by the SIMO scheme.

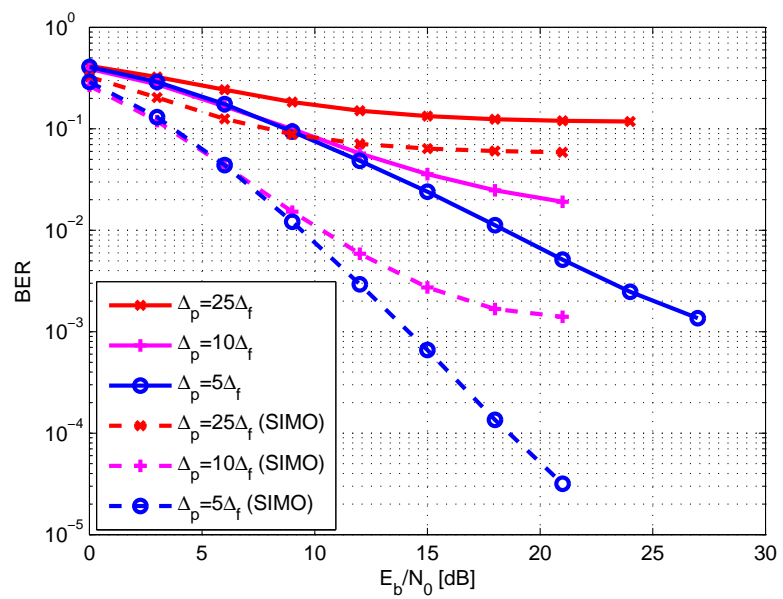

Fig. 8. Parking scenario with $\mathrm{K}=-10 \mathrm{~dB}$. Performance evaluation with the introduction of antenna diversity (SIMO) and with the reduction of the pilot sub-carrier spacing $\left(\Delta_{p}=5\right.$ and $\left.10 \Delta_{f}\right)$ respect to the standard specification case $\left(\Delta_{p}=25 \Delta_{f}\right)$.

\section{CONCLUSION}

In this paper we presented an analysis of the FL based on the OFDM mode of the IEEE 802.16e standard over a novel realistic airport channel model. We provided performance for four different airport scenarios, showing that the most critical scenarios are those with large delays and NLOS conditions. Given the system characteristics, and more specifically the sub-carrier spacing, the impact of typical Doppler spread/shift on the performance is negligible. Vice versa, the values of delay spread that are typical for some airport environment together with the large pilot sub-carrier spacing represent the main critical point, jointly with the lack of time diversity. We investigated a performance enhancement through the addition of a second antenna at the receiver side (SIMO) and the reduction of the pilot sub-carrier distance, applying it to the parking scenario. For our investigation the OFDM mode of the WiMAX standard may be suitable for the airport FL data-link, nevertheless, especially for NLOS scenarios, improvements as the ones proposed herein have to be accounted.

\section{ACKNOWLEDGEMENTS}

We wish to thank the colleagues of the DLR institute of Communication and Navigation Dr. Michael Schnell, Dr. Snjezana Gligorevic, Dr. Sinja Brandes, Dr. Gianluigi Liva and Prof. Marco Chiani from the Bologna University, for useful discussions and suggestions.

\section{REFERENCES}

[1] RTCA, "Signal-In-Space Minimum Aviation System Performance Standards (MASPS) for Advanced VHF Digital Data Communications including compatibility with Digital Voice Techniques," Sep. 2000.

[2] Air Navigation Commission, "Report on the Results of the ITU World Radiocommunication Conference (WRC-07)," 2007.

[3] IEEE 802.16-2005, "Part 16: Air Interface for Fixed and Mobile Broadband Wireless Access Systems, Amendment 2: Physical and Medium Access Control Layers for Combined Fixed and Mobile Operation in Licensed Bands and Corrigendum 1," Feb. 2006.

[4] IEEE 802.16-2004, "Local and Metropolitan Area Networks Part 16 Air Interface for Fixed Broadband Wireless Access Systems," Oct. 2004.

[5] S. Gligorevic, R. Zierhut, T. Jost, W. Wang, "Airport Channel Measurement at 5.2 GHz." EuCAP, Mar. 2009.

[6] P. A. Bello, "Characterization of randomly time-variant linear channels," IEEE Trans. Comm., vol. 11, no. 4, pp. 360-393, Dec. 1963.

[7] R. H. Clarke, "A statistical theory of mobile-radio reception," Bell Sys. Tech. J., vol. 47, no. 6, pp. 957-1000, July/Aug. 1968.

[8] S. O. Rice, "Mathematical analysis of random noise," Bell Sys. Tech. J., vol. 23, pp. 282-332, July 1944

[9] —- "Mathematical analysis of random noise," Bell Sys. Tech. J., vol. 24, pp. 46-156, Jan. 1945.

[10] P. Höher, "Kohärenter Empfang trelliscodierter PSK-Signale auf frequenzselektiven Mobilfunkkanälen; Entzerrung, Decodierung und Kanalparameterschätzung," vol. 147, 1990.

[11] _ , "A statistical discrete-time model for the wssus multipath channel," vol. 41, no. 4. IEEE Trans. Veh. Technol., Nov. 1992, pp. 461-468.

[12] H. Schulze, "Stochastische Modelle und digitale Simulation von Mobilfunkkanälen," vol. 32, pp. 473-483, 1989.

[13] P. A. Bello, "Aeronautical channel characterization," IEEE Trans. Inform. Theory, vol. 21, pp. 548-563, May 1973. 